\title{
Third degree heart block and elevated troponin following acute respiratory hypoxic failure in a COVID-19 recovered patient in the absence of coronary artery disease
}

\author{
Samer Khalil ${ }^{1}$, Andrew Collins ${ }^{2}$, Naser Mubarak ${ }^{2 *}$ and Taher Khalil ${ }^{3}$ \\ ${ }^{1}$ Edward Via College of Osteopathic Medicine, USA \\ ${ }^{2}$ University of Central Florida College of Medicine, USA \\ ${ }^{3}$ Advent Health Medical Group, USA
}

\begin{abstract}
A 53-year-old female recently recovered from COVID-19 was re-admitted to the ICU with third degree heart block and acute respiratory hypoxic failure with no evidence of coronary artery disease following a left heart catheterization. The patient was placed on a sequential AV pacemaker and demonstrated clinical improvement.
\end{abstract}

\section{Introduction}

Beginning in December 2019, COVID-19 has extensively escalated to more than 23 million confirmed cases worldwide and over 5 million within the United States alone. Most in-hospital clinical diagnoses are based on a nasal swab culture and presentation of significant symptoms including dyspnea, fatigue, fever/chills, cough-these 'typical' diagnostic symptoms are inherent to respiratory pathology and mimic viral pneumonia. Albeit cardiovascular tissue pathology has been associated with more severe cases and deteriorating patient outcomes including Intensive Care Unit (ICU) admission and increased inhospital patient mortality [1,2].

Elevated troponin levels are a key diagnostic laboratory marker to specify myocardial injury $[1,3]$.There have been several hypotheses on the pathophysiology behind COVID-19 associated myocardial injury, many of which suggest the ACE- 2 receptor as inherent to inception of disease [4]. To date, these myocardial pathological associations with COVID-19 patients have been discussed: microangiopathy, myocarditis, myocardial infarction (in those with atherosclerotic risk factors and previous cardiovascular disease), and cytokine storm. The ACE-2 receptor is the primary binding site for SARS-CoV-2 cellular entry, highly expressed within the adult human heart; furthermore, the virus seems to downregulate expression of ACE-2 once infection has proceeded, therefore reducing the conversion of Angiotensin-II (Ang-II) to Angiotensin 1-7 (Ang 1-7) [5]. Ang 1-7 has a protective physiological role for the heart and other target organs, thus reduced levels could potentially lead to myocardial injury and elevated troponin levels [6].

We describe a patient who initially admitted to the hospital with acute respiratory hypoxic failure associated with a positive nasopharyngeal swab for COVID-19. Twelve days later, the patient presented to the emergency department with third degree heart block and was placed on an external pacer. The patient was then admitted to cardiology for temporary pacemaker placement. Three days later, within the ICU, the patient had marked increase in cardiac troponin levels and received a left heart catheterization coronary angiography. Remarkably, angiography results reported no significant coronary artery disease.

\section{Learning objectives}

- To recognize and identify abnormal signs of myocardial injury in COVID-19 infected patients.

- To understand the mechanisms responsible for COVID-19 related elevated cardiac-specific troponin.

\section{History of present illness}

A 53-year old female was admitted to the ICU for critical care management following EVAC and ER findings of a complex bradycardic rhythm with a reported rate in the 20 s and acute hypoxic respiratory failure. On presentation to the hospital, the patient presented with fatigue and a dysconjugate gaze. The patient had been recently admitted in our services for acute hypoxic respiratory failure associated with COVID-19. Plaquenil was not started at the time due to a slightly prolonged QT of 422. She reported experiencing fatigue and difficulty completing ADLs since that time due to feeling tired.

*Correspondence to: Naser Mubarak, Medical Student at University of Central Florida College of Medicine and the Center for Public Health Initiatives at the University of Pennsylvania, 6850 Lake Nona Blvd. Orlando, FL. 32824 UCF College of Medicine, USA, Tel: (386)-898-3748, E-mail: Nmubarak94@knights.ucf.edu

Key words: COVID-19, coronavirus, troponin, myocardial injury, myocardial infarction, AV Block

Received: October 05, 2020; Accepted: October 13, 2020; Published: October 21,2020 


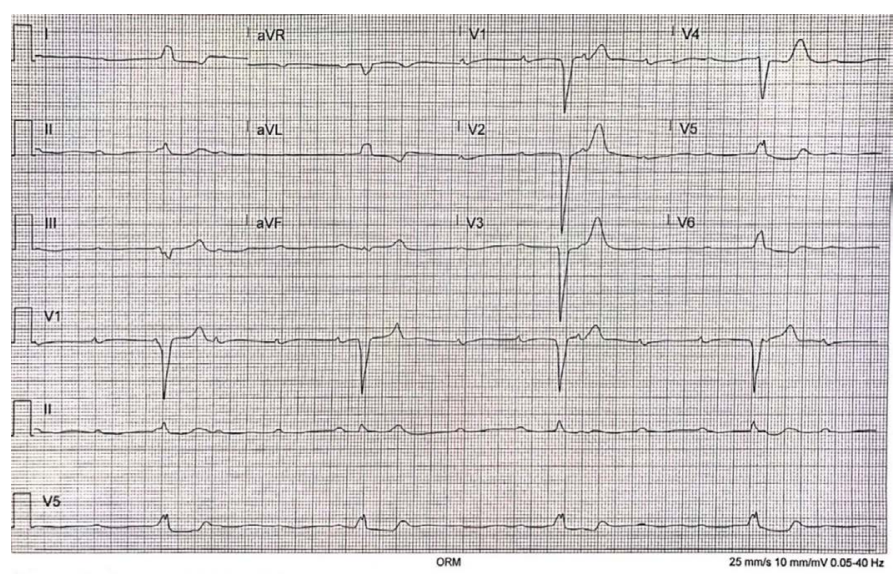

Figure 1. EKG Demonstrating Complete AV Block: Complete absence of AV noda conduction with increased risk ventricular standstill and sudden cardiac death. Required urgent admission for cardiac monitoring, backup temporary pacing and later insertion of a permanent pacemaker

\section{Differential diagnosis}

Given the initial presentation, ECG findings, and marked troponin levels, a wide differential was considered including complete heart block, acute coronary syndrome, and pulmonary embolism.

\section{Past medical history}

The patient had a history of end-stage-renal disease on peritoneal dialysis, anemia of chronic disease, diabetes mellitus, hypertension, and a recent SARS-COV2 admission with severe septic shock and received convalescent transfusion. The patient also had acute nontraumatic rhabdomyolysis during that admission. The patient was taking aerosol albuterol, $90 \mathrm{mcg} /$ ing 1 puff 4 times daily, amlodipine, $10 \mathrm{mg}$ oral daily, Auryxia, $210 \mathrm{mg}$ oral 3 times daily with meals, b-12, $1000 \mathrm{mcg}$ oral tablet, benzonatate, $100 \mathrm{mg}$, calcitriol, $0.5 \mathrm{mcg}$ oral, cinacalcet, 30 $\mathrm{mg}$ oral daily, dapsone, $25 \mathrm{mg}$ oral daily, doxazosin, $2 \mathrm{mg}$ at bedtime, doxycycline hyclate $100 \mathrm{mg}$ daily, Eliquis, $5 \mathrm{mg}$ daily, gentamicin, $0.1 \%$ topical cream, hydralazine, $50 \mathrm{mg}$ oral 3 times a day, metoprolol succinate ER $100 \mathrm{mg}$ daily, sevelamer carbonate, $800 \mathrm{mg}$ daily.

\section{Investigations}

EKG displayed significant prolonged QT of 632 and complete AV heart block. CXR showed vascular congestion with increased interstitial markings bilaterally, cardiomegaly, and trace bilateral pleural effusions. Transthoracic echocardiogram indicated a left ventricular EF of 60-65 percent, mild left atrial dilation, mild to moderate AP and MT valvular regurgitation, pericardial effusion, and a dilated ICV with less than 50 percent respiratory change in the IVC dimension. Labs reported elevated troponin $(6735 \mathrm{ng} / \mathrm{L})$, metabolic acidosis secondary to lactic acidosis $(3.7 \mathrm{mmol} / \mathrm{L})$, chronic anemia (RBC, $\left.2.53 \times 10^{6} / \mathrm{microL}\right)$, elevated BUN (111 mg/dL), mild leukocytosis $\left(11.6 \times 10^{3} / \mathrm{microL}\right)$, and elevated liver enzymes (AST 188 units/L and ALT 41 Units/L). Left heart catheterization coronary angiogram demonstrated no significant coronary artery disease.

\section{Management}

On the basis of the patient's symptoms on presentation and their history of COVID-19, the patient was placed on temporary transcutaneous pacing of 120 milliamps for capture and an isoproterenol infusion. The patient continued her peritoneal dialysis per nephrology service recommendation. The patient's respiratory failure improved with fluid removal via patient dialysis and she was weaned down to room air. The patient was also removed from vasopressors. During her stay in the ICU, she underwent a left heart catheterization coronary angiogram in response a marked increase in troponin followed by placement of an AV sequential pacemaker.

\section{Discussion}

Elevated troponin and complete AV block in the absence of coronary artery disease. Third degree AV block is most commonly associated with pathologic findings, such CAD or recent infection, drug intoxication, or post-surgical intervention [7]. Without a reversible and identifiable etiology of the complete heart block, permanent pacemaker placement has been associated with positive patient outcomes [8]. Patient outcomes with ischemic versus non-ischemic complete heart block have not been shown to have statistically significant differences in mortality [9].

Our patient presented with elevated cardiac-specific troponins as well. While often indicative of cardiac diseases and interventions, such as cardiac surgery, heart failure, and myocarditis, the non-cardiac diseases of renal failure, sepsis, and critical illness, may also elevate troponins [10]. Her catheterization and pacemaker placement may have caused the marked increase in troponins, however, her history of chronic kidney disease and recent SARS-CoV-2 pneumonia may have led to further elevation.

While many health complications and underlying diseases can cause increased mortality associated with SARS-CoV-2 infection, hospitalized patients with CVD have shown a nearly $20 \%$ overall mortality when presenting with COVID-19 [2]. Furthermore, in-hospital mortality has been shown to reach as high $59.6 \%$ in cases with laboratory-confirmed COVID-19 and elevated troponin levels, compared to $8.9 \%$ in those with normal troponin levels [11].

Although mechanistically unclear, it is hypothesized that the COVID-19 and other viral infections can lead to an increased vascular cellular inflammation, myocardial oxygen demand, proinflammatory effects, plaque rupture, and decreased myocardial oxygen supply, all leading to increased risk of heart block, myocardial infarction, heart failure, and arrythmia [12,13]. Involvement of the cardiovascular system through renin-angiotensin-aldosterone system mediated via ACE2 receptor depletion has also been noted [14]. Such acute coronary events could result from increased myocardial demand triggered by infections precipitating myocardial injury or infarction [11]. Primarily, the definition of myocardial injury associated with COVID-19 is based on elevated serum levels of cardiac-specific troponins, however these values are highly variable. This myocardial injury presenting with elevated cardiac-troponin levels has been associated with arrythmia and left ventricular systolic dysfunction [1,2].

\section{Follow-up}

The patient recovered well and was discharged with scheduled cardiology follow-up.

\section{Conclusion}

A 53-year-old-female who originally presented with complex bradycardic rhythm and acute hypoxic respiratory failure, experienced elevated cardiac-specific troponins, third degree AV block, and nonSTEMI following recent recovery from SARS-CoV-2 pneumonia. The specific mechanisms remain speculative for cardiac involvement of COVID-19; however, it is observed that underlying cardiac disease in the infected patients is associated with poor clinical outcomes, including 
Mubarak N (2021) Third degree heart block and elevated troponin following acute respiratory hypoxic failure in a COVID-19 recovered patient in the absence of coronary artery disease

high mortality. Combined elevated troponin and laboratory-confirmed COVID-19 is associated with nearly $60 \%$ in-hospital mortality. Although patients with COVID-19 initially present as primarily a respiratory condition, imbalances of the renin-angiotensin-aldosterone system via ACE-2 receptor depletion likely leads to involvement of involvement of the cardiovascular system. Subsequent acute coronary events, such as myocardial infarction, heart failure, and arrythmia have been noted to follow.

\section{Conflict of interest}

The authors report no conflicts of interest in this work.

\section{References}

1. Tao G, Fan Y, Chen M, Wu X, Zhang L, et al. (2020) Cardiovascular Implications of Fatal Outcomes of Patients with Coronavirus Disease 2019 (COVID-19). JAMA Cardiol 5: 811-818. [Crossref]

2. Shaobo S, Qin M, Shen B, Cai Y, Liu T, et al. (2020) Association of Cardiac Injury with Mortality in Hospitalized Patients With COVID-19 in Wuhan, China. JAMA Cardiol 5: 802-810 [Crossref]

3. Long B, Long DA, Tannenbaum L, Koyfman A (2020) An emergency medicine approach to troponin elevation due to causes other than occlusion myocardial infarction. Am J Emerg Med 38: 998-1006 [Crossref]

4. Chen L, Li X, Chen M, Feng Y, Xiong C (2020) The ACE2 expression in human heart indicates new potential mechanism of heart injury among patients infected with SARSCoV-2. Cardiovasc Res 116: 1097-1100.

5. Patel VB, Zhong JC, Grant MB, Oudit GY (2016) Role of the ACE2/angiotensin 1-7 axis of the renin-angiotensin system in heart failure. Circ Res 118:1313-1326. [Crossref]
6. Vaduganathan M, Vardeny O, Michel T, McMurray JJV, Pfeffer MA, et al. (2020) Renin-angiotensin-aldosterone system inhibitors in patients with Covid-19. $N$ Engl $J$ Med 382:1653-1659. [Crossref]

7. Zoob M, Smith KS (1963) Aetiology of Complete Heart-block. Br Med J 2: 1149-1153. [Crossref]

8. Kusumoto FM, Schoenfeld MH, Barrett C, Edgerton JR, Ellenbogen KA, et al. (2019) 2018 ACC/AHA/HRS Guideline on the Evaluation and Management of Patients with Bradycardia and Cardiac Conduction Delay: Executive Summary: A Report of the American College of Cardiology/American Heart Association Task Force on Clinical Practice Guidelines, and the Heart Rhythm Society. J Am Coll Cardiol 74: 932-987. [Crossref]

9. Sundhu M, Yildiz M, Syed M, Shah B, Gul S, et al. (2017) Clinical Characteristics and Outcomes of Patients with Ischemic and Non-Ischemic Complete Heart Block. Cureus 9: e1244.[Crossref]

10. Ammann P, Pfisterer M, Fehr T, Rickli H (2004) Raised cardiac troponins. BMJ 328 1028-1029. [Crossref]

11. Bonow RO, Fonarow GC, Gara P, Yancy CW (2020) Association of Coronavirus Disease 2019 (COVID-19) With Myocardial Injury and Mortality. JAMA Cardiol 5: 751-753 [Crossref]

12. Madjid M, Safavi-Naeini P, Solomon SD, Vardeny O (2020) Potential Effects of Coronaviruses on the Cardiovascular System: A Review. JAMA Cardiol 5: 831-840 [Crossref]

13. Clerkin KJ, Fried JA, Raikhelkar J, Sayer G, Griffin JM, et al. (2020) COVID-19 and Cardiovascular Disease. Circulation 141: 1648-1655. [Crossref]

14. Tersalvi G, Vicenzi M, Calabretta D, Biasco L, Pedrazzini G, et al. (2020) Elevated Troponin in Patients with Coronavirus Disease 2019: Possible Mechanisms. J Card Fail 26: 470-475. [Crossref]

Copyright: (C2021 Mubarak N. This is an open-access article distributed under the terms of the Creative Commons Attribution License, which permits unrestricted use, distribution, and reproduction in any medium, provided the original author and source are credited. 